\title{
BOUNDS FOR KAKEYA-TYPE MAXIMAL OPERATORS ASSOCIATED WITH $k$-PLANES
}

\author{
RICHARD OBERLIN
}

\begin{abstract}
A $(d, k)$ set is a subset of $\mathbb{R}^{d}$ containing a translate of every $k$-dimensional plane. Bourgain showed that for $k \geq k_{c r}(d)$, where $k_{c r}(d)$ solves $2^{k_{c r}-1}+k_{c r}=d$, every $(d, k)$ set has positive Lebesgue measure. We give a short proof of this result which allows for an improved $L^{p}$ estimate of the corresponding maximal operator, and which demonstrates that a lower value of $k_{c r}$ could be obtained if improved mixed-norm estimates for the $x$-ray transform were known.
\end{abstract}

\section{Introduction}

A measurable set $E \subset \mathbb{R}^{d}$ is said to be a $(d, k)$ set if it contains a translate of every $k$-dimensional plane in $\mathbb{R}^{d}$. Once the definition is given, the question of the minimum size of a $(d, k)$ set arises. This question has been extensively studied for the case $k=1$, the Kakeya sets. It is known that there exist Kakeya sets of measure zero, and these are called Besicovitch sets. It is conjectured that all Besicovitch sets have Hausdorff dimension $d$. For $k \geq 2$, it is conjectured that $(d, k)$ sets must have positive measure, i.e. that there are no $(d, k)$ Besicovitch sets. These size estimates are related to $L^{p}$ bounds on two maximal operators which we define below.

Let $G(d, k)$ denote the Grassmannian manifold of $k$-dimensional linear subspaces of $\mathbb{R}^{d}$. For $L \in G(d, k)$ we define

$$
\mathcal{N}^{k}[f](L)=\sup _{x \in \mathbb{R}^{d}} \int_{x+L} f(y) d y
$$

where we will only consider functions $f$ supported on the unit ball $B(0,1) \subset \mathbb{R}^{d}$.

A limiting and rescaling argument shows that if $\mathcal{N}^{k}$ is bounded for some $p<\infty$ from $L^{p}\left(\mathbb{R}^{d}\right)$ to $L^{1}(G(d, k))$, then $(d, k)$ sets must have positive measure. By testing $\mathcal{N}^{k}$ on the characteristic function of $B(0, \delta), \chi_{B(0, \delta)}$, one sees that such a bound may only hold for $p \geq \frac{d}{k}$. For $L$ in $G(d, k)$ and $a \in \mathbb{R}^{d}$ define the $\delta$ plate centered at $a, L_{\delta}(a)$, to be the $\delta$ neighborhood in $\mathbb{R}^{d}$ of the intersection of $B\left(a, \frac{1}{2}\right)$ with $L+a$. Fixing $L$, considering $\mathcal{N}^{k} \chi_{L_{\delta}(0)}$, and using the fact that the dimension of $G(d, k)$ is $k(d-k)$, we see that a bound into $L^{q}(G(d, k))$ can only hold for $q \leq k p$. This leads to the following conjecture, where the case $k=1$ is excluded due to the existence of Besicovitch sets.

Conjecture 1.1. For $2 \leq k<d, p>\frac{d}{k}, 1 \leq q \leq k p$

$$
\left\|\mathcal{N}^{k} f\right\|_{L^{q}(G(d, k))} \lesssim\|f\|_{L^{p}\left(\mathbb{R}^{d}\right)} .
$$

Received by the editors December 15, 2005.

2000 Mathematics Subject Classification. 42B25. 
It is also useful to consider a generalization of the Kakeya maximal operator, defined for $L \in G(d, k)$ by

$$
\mathcal{M}_{\delta}^{k}[f](L)=\sup _{a \in \mathbb{R}^{d}} \frac{1}{\mathcal{L}^{d}\left(L_{\delta}(a)\right)} \int_{L_{\delta}(a)} f(y) d y
$$

where $\mathcal{L}^{d}$ denotes Lebesgue measure on $\mathbb{R}^{d}$. Using an argument analogous to that in Lemma 2.15 of [2], one may see that a bound

$$
\left\|\mathcal{M}_{\delta}^{k} f\right\|_{L^{1}(G(d, k))} \lesssim \delta^{\frac{-\alpha}{p}}\|f\|_{L^{p}\left(\mathbb{R}^{d}\right)}
$$

where $\alpha>0$ and $p<\infty$, implies that the Hausdorff dimension of any $(d, k)$ set is at least $d-\alpha$. Considering $\mathcal{M}_{\delta}^{k} \chi_{B(0, \delta)}$ and $\mathcal{M}_{\delta}^{k} \chi_{L_{\delta}(0)}$, we formulate

Conjecture 1.2. For $k \geq 1, p<\frac{d}{k}, q \leq(d-k) p^{\prime}$

$$
\left\|\mathcal{M}_{\delta}^{k} f\right\|_{L^{q}(G(d, k))} \lesssim \delta^{k-\frac{d}{p}}\|f\|_{L^{p}\left(\mathbb{R}^{d}\right)} .
$$

In [6] Falconer showed that, for any $\epsilon>0, \mathcal{N}^{k}$ is bounded from $L^{\frac{d}{k}+\epsilon}\left(\mathbb{R}^{d}\right)$ to $L^{1}(G(d, k))$ when $k>\frac{d}{2}$. Later, in [2], Bourgain used a Kakeya maximal operator bound combined with an $L^{2}$ estimate of the $x$-ray transform to show that $\mathcal{N}^{k}$ is bounded from $L^{p}\left(\mathbb{R}^{d}\right)$ to $L^{p}(G(d, k))$ for $(d, k, p)=(4,2,2+\epsilon)$ and $(d, k, p)=(7,3,3+$ $\epsilon)$. He then showed, using a recursive metric entropy estimate, that for $d \leq 2^{k-1}+k$, $\mathcal{N}^{k}$ is bounded for a large unspecified $p$. Substituting in the proof Katz and Tao's more recent bound for the Kakeya maximal operator from [7]

$$
\left\|\mathcal{M}_{\delta}^{1} f\right\|_{L^{n+\frac{3}{4}}(G(n, 1))} \lesssim \delta^{-\left(\frac{3(n-1)}{4 n+3}+\epsilon\right)}\|f\|_{L^{\frac{4 n+3}{7}}\left(\mathbb{R}^{n}\right)}
$$

one now sees that this holds for $k>k_{c r}(d)$ where

$$
k_{c r}(d) \text { solves } d=\frac{7}{3} 2^{k_{c r}-2}+k_{c r} .
$$

By Hölder's inequality, the following is true for any $k$-plate $L_{\delta}$ and positive $f$

$$
\int_{L_{\delta}} f d x \lesssim \delta^{\frac{d-k}{r^{\prime}}}\left(\int_{L^{\perp}}\left(\int_{L+y} f(x) d \mathcal{L}^{k}(x)\right)^{r} d \mathcal{L}^{d-k}(y)\right)^{\frac{1}{r}} .
$$

Combining this with the $L^{p} \rightarrow L^{q}\left(L^{r}\right)$ bounds for the $k$-plane transform which were proven by Christ in Theorem A of [4], we see that Conjecture 1.2 holds when $p \leq \frac{d+1}{k+1}$. Except for a factor of $\delta^{-\epsilon}$, the same bound for $\mathcal{M}_{\delta}^{k}$ was proven with $k=2$ by Alvarez in [1] using a geometric-combinatorial "bush"-type argument. Alvarez also used a "hairbrush" argument to show that $(d, 2)$ sets have Minkowski dimension at least $\frac{2 d+3}{3}$. More recently, Mitsis proved a similar maximal operator bound in [11] and showed that $(d, 2)$ sets have Hausdorff dimension at least $\frac{2 d+3}{3}$ in [10]. In [3], Bueti used a hairbush type argument to show that, in the setting of vector spaces over finite fields, Conjecture 1.2 holds when $p \leq \frac{d+1+\frac{1}{k}}{k+1}$ and $k<d-1$. In [13], Rogers gave estimates for the Hausdorff dimension of sets which contain planes in directions corresponding to certain curved submanifolds of $G(4,2)$.

Our main result is the following. 
Theorem 1.1. Suppose $4 \leq k<d$ and $k>k_{c r}(d)$, where $k_{c r}(d)$ is defined in (3). Then

$$
\left\|\mathcal{N}^{k} f\right\|_{L^{p}(G(d, k))} \lesssim\|f\|_{L^{p}\left(\mathbb{R}^{d}\right)}
$$

for $f$ supported on the unit ball and $p \geq \frac{d-1}{2}$. If, additionally, we have $k-j>k_{c r}(d-j)$ for some integer $j$ in $[1, k-4]$, then we may take $p \geq \frac{d-1}{2+j}$.

For $k<k_{c r}(d)$, we do not have a bound for $\mathcal{N}^{k}$, however our technique yields certain bounds for $\mathcal{M}_{\delta}^{k}$.

Theorem 1.2.

$$
\left\|\mathcal{M}_{\delta}^{k} f\right\|_{L^{q}(G(d, k))} \lesssim \delta^{-\frac{\alpha}{p}}\|f\|_{L^{p}\left(\mathbb{R}^{d}\right)}
$$

when

$$
k \geq 2, \alpha=d-k p+\epsilon, p=\frac{d}{k+\frac{3}{4}}, q \leq(d-k)\left(\frac{4(d-(k-1))}{7}\right)^{\prime}
$$

or

$$
k \geq 2, \alpha=\frac{3(d-k)}{7\left(2^{k-1}\right)}+\epsilon, p=\frac{d+1}{2}, q=d+1
$$

or

$$
3 \leq k \leq k_{c r}(d), \alpha=\frac{3(d-k)}{7\left(2^{k-2}\right)}-1+\epsilon, p=q=\frac{d}{2}
$$

where $\epsilon>0$ may be taken arbitrarily small.

In (5) we have an optimal value for $p$ relative to $\alpha$, but a non-optimal value for $q$. In (6) and (7) we have improved values of $\alpha$ at the cost of a non-optimal $p$. For the "non-borderline" $k$, specifically when $k+1<k_{c r}(d+1)$, (6) gives a smaller value of $\alpha$ than (7).

The number $p=\frac{d-1}{2+j}$ in Theorem 1.1 and the number $p=\frac{d}{k+\frac{3}{4}}$ in Theorem 1.2 are approximate and may be slightly improved through careful numerology. Also, in (7) we may take $k=2$, but a slightly higher value of $p$ and $q$ is then required.

We prove (5) and (6) in Section 2 through a recursive maximal operator bound which is derived using Drury and Christ's bounds for the $x$-ray transform and which is inspired by Bourgain's recursive metric entropy estimates. This recursive maximal operator bound is a slight improvement of the result in [12], which will remain unpublished, and the new bound comes with a vastly simplified proof afforded by the explicit use of the $x$-ray transform. Additionally our argument reveals that with certain adjustments of $p$ and $q$, the number 2 in the definition of $k_{c r}(d)$ and in the definition of $\alpha$ in (6) and (7) may be replaced by the ratio $\frac{\tilde{r}}{\tilde{p}}$ if the $x$-ray transform is known to be bounded, for certain values of $n$, from $L^{p_{n}}\left(\mathbb{R}^{n}\right)$ to $L_{\mathbb{S}^{n-1}}^{q_{n}}\left(L_{\mathbb{R}^{n-1}}^{r_{n}}\right)$ for any $r_{n}, p_{n}, q_{n}$ satisfying $\frac{r_{n}}{p_{n}}=\frac{\tilde{r}}{\tilde{p}}$.

We prove (7) and Theorem 1.1 in Section 3. There, we combine (5) and (6) with the $L^{2}$ method which Bourgain used to give bounds for $\mathcal{N}^{k}$ when $(d, k)=(4,2)$ or $(7,3)$.

From (6) and (7) we see that, for $k \geq 2$, the Hausdorff dimension of any $(d, k)$ set is at least

$$
\min \left(d, \max \left(d-\frac{3(d-k)}{7\left(2^{k-2}\right)}+1, d-\frac{3(d-k)}{7\left(2^{k-1}\right)}\right)\right) .
$$


When $(d-k)<7$, it is preferable to start with Wolff's $L^{\frac{n+2}{2}}$ bound for the Kakeya maximal operator from [15], instead of (2). A similar procedure then gives the lower bound

$$
\min \left(d, \max \left(d-\frac{d-k-1}{2^{k-1}}+1, d-\frac{d-k-1}{2^{k}}\right)\right)
$$

for the Hausdorff dimension of a $(d, k)$ set.

It should be noted that the dimension estimates provided by applying (6) and it's Wolff-variant are also a direct consequence of the metric entropy estimates in [2]. However, to the best of the author's knowledge they have not previously appeared in the literature, even without the improvement obtained from [16] and [7].

\section{A recursive maximal operator bound}

We start with the definition of the measure we will use on $G(d, k)$. Fix any $L \in$ $G(d, k)$. For a Borel subset $F$ of $G(d, k)$ let

$$
\mathcal{G}^{(d, k)}(F)=\mathcal{O}(\{\theta \in O(d): \theta(L) \in F\})
$$

where $\mathcal{O}$ is normalized Haar measure of the orthogonal group on $\mathbb{R}^{d}, O(d)$. By the transitivity of the action of $O(d)$ on $G(d, k)$ and the invariance of $\mathcal{O}$, it is clear that the definition is independent of the choice of $L$. Also note that $\mathcal{G}^{(d, k)}$ is invariant under the action of $O(d)$. By the uniqueness of uniformly-distributed measures (see [9], pages 44-53), $\mathcal{G}^{(d, k)}$ is the unique normalized Radon measure on $G(d, k)$ invariant under $O(d)$.

It will be necessary to use an alternate formulation of $\mathcal{G}^{(d, k)}$. For each $\xi$ in $\mathbb{S}^{d-1}$ let $T_{\xi}: \xi^{\perp} \rightarrow \mathbb{R}^{d-1}$ be an orthogonal linear transformation. Then $T_{\xi}^{-1}$ identifies $G(d-1, k-1)$ with the $k-1$ dimensional subspaces of $\xi^{\perp}$. Now, define $T: \mathbb{S}^{d-1} \times$ $G(d-1, k-1) \rightarrow G(d, k)$ by

$$
T(\xi, M)=\operatorname{span}\left(\xi, T_{\xi}^{-1}(M)\right) .
$$

Choosing $T_{\xi}$ continuously on the upper and lower hemispheres of $\mathbb{S}^{d-1}, T^{-1}$ identifies the Borel subsets of $G(d, k)$ with the completion of the Borel subsets of $\mathbb{S}^{d-1} \times G(d-$ $1, k-1)$. Under this identification, by uniqueness of rotation invariant measure, we have

$$
\mathcal{G}^{(d, k)}(F)=\sigma^{d-1} \times \mathcal{G}^{(d-1, k-1)}\left(T^{-1}(F)\right)
$$

where $\sigma^{d-1}$ denotes normalized surface measure on the unit sphere.

For a function $f$ on $\mathbb{R}^{d}, \xi \in \mathbb{S}^{d-1}$, and $y \in \xi^{\perp}$, the $x$-ray transform of $f$ is defined

$$
f_{\xi}(y)=\int_{\mathbb{R}} f(y+t \xi) d t .
$$

It is conjectured that the $x$-ray transform is bounded from $L^{p}\left(\mathbb{R}^{d}\right)$ to $L_{\mathbb{S}^{d-1}}^{q}\left(L_{\mathbb{R}^{d-1}}^{r}\right)$ when $p, q, r$ satisfy

$$
\begin{aligned}
r & <\infty \\
p & =\frac{r d}{d+r-1} \\
q & \leq r^{\prime} d .
\end{aligned}
$$


This was shown to hold in [5] for $p<\frac{d+1}{2}$ and in [4] for $p=\frac{d+1}{2}$. Also, see [16] and [8] for certain improvements.

In the following proposition we exploit the fact that $r>p$ when $r \neq 1$ in (9), i.e. that the $x$-ray transform is $L^{p}$-improving.

Proposition 2.1. Suppose that $p \leq d+1$ and $k \geq 2$. Then a bound

$$
\left\|\mathcal{M}_{\delta}^{k-1} f\right\|_{L^{q}(G(d-1, k-1))} \lesssim \delta^{-\frac{\alpha}{p}}\|f\|_{L^{p}\left(\mathbb{R}^{d-1}\right)}
$$

for all $f \in L^{p}\left(\mathbb{R}^{d-1}\right)$ implies the bound

$$
\left\|\mathcal{M}_{\delta}^{k} f\right\|_{L^{\tilde{q}}(G(d, k))} \lesssim \delta^{-\frac{\tilde{\tilde{p}}}{\tilde{p}}}\|f\|_{L^{\tilde{p}}\left(\mathbb{R}^{d}\right)}
$$

for all $f \in L^{\tilde{p}}\left(\mathbb{R}^{d}\right)$ with

$$
\tilde{p}=p \frac{d}{d+p-1}, \quad \tilde{\alpha}=\alpha \frac{\tilde{p}}{p}=\alpha \frac{d}{d+p-1}, \quad \text { and } \quad \tilde{q}=\min \left(q, d p^{\prime}\right) .
$$

Proof. Without loss of generality, we assume that $f$ is positive. Let $L \in G(d, k)$ and suppose that $L=\operatorname{span}\left(\xi, T_{\xi}^{-1}(M)\right)$ where $M \in G(d-1, k-1)$. Let $a_{L} \in \mathbb{R}^{d}$ and let $a_{M}=T_{\xi}\left(\operatorname{proj}_{\xi^{\perp}}\left(a_{L}\right)\right)$, where proj denotes orthogonal projection. Then

$$
\begin{array}{r}
\int_{L_{\delta}\left(a_{L}\right)} f(y) d y \leq \int_{M_{\delta}\left(a_{M}\right)} \int_{\mathbb{R}} f\left(T_{\xi}^{-1}(x)+t \xi\right) d t d x \\
=\int_{M_{\delta}\left(a_{M}\right)} f_{\xi}\left(T_{\xi}^{-1}(x)\right) d x
\end{array}
$$

where $L_{\delta}\left(a_{L}\right)$ and $M_{\delta}\left(a_{M}\right)$ are $k$ and $k-1$ plates respectively. Noting that $d-k=$ $(d-1)-(k-1)$, it follows that

$$
\mathcal{M}_{\delta}^{k}[f](L) \lesssim \mathcal{M}_{\delta}^{k-1}\left[f_{\xi} \circ T_{\xi}^{-1}\right](M) .
$$

By (8), Hölder's inequality, and our hypothesized bound, we now have

$$
\begin{array}{r}
\left\|\mathcal{M}_{\delta}^{k}[f]\right\|_{L^{\tilde{q}}(G(d, k))} \lesssim\left(\int_{\mathbb{S}^{d-1}} \int_{G(d-1, k-1)} \mathcal{M}_{\delta}^{k-1}\left[f_{\xi} \circ T_{\xi}^{-1}\right](M)^{\tilde{q}} d M d \xi\right)^{\frac{1}{\tilde{q}}} \\
\lesssim\left(\int_{\mathbb{S}^{d-1}}\left(\int_{G(d-1, k-1)} \mathcal{M}_{\delta}^{k-1}\left[f_{\xi} \circ T_{\xi}^{-1}\right](M)^{q} d M\right)^{\frac{\tilde{q}}{q}} d \xi\right)^{\frac{1}{\tilde{q}}} \\
\lesssim \delta^{-\frac{\alpha}{p}}\left(\int_{\mathbb{S}^{d-1}}\left(\int_{\mathbb{R}^{d-1}}\left(f_{\xi} \circ T_{\xi}^{-1}(x)\right)^{p} d x\right)^{\frac{\tilde{q}}{p}} d \xi\right)^{\frac{1}{\tilde{q}}} \\
=\delta^{-\frac{\alpha}{p}}\left(\int_{\mathbb{S}^{d-1}}\left(\int_{\xi^{\perp}} f_{\xi}(x)^{p} d x\right)^{\frac{\tilde{q}}{p}} d \xi\right)^{\frac{1}{\tilde{q}}} .
\end{array}
$$

Finally, by our restrictions on $p$ and $\tilde{q}$, we may apply Drury and Christ's bound for the $x$-ray transform, obtaining

$$
\left(\int_{\mathbb{S}^{d-1}}\left(\int_{\xi^{\perp}} f_{\xi}(x)^{p} d x\right)^{\frac{\tilde{q}}{p}} d \xi\right)^{\frac{1}{\tilde{q}}} \lesssim\|f\|_{L^{\tilde{p}}\left(\mathbb{R}^{d}\right)}
$$


when $\tilde{p}=\frac{p d}{d+p-1}$.

One should note that if $\alpha=(d-1)-(k-1) p$, then $\tilde{\alpha}=d-k \tilde{p}$. Hence, except for a non-optimal $\tilde{q}$, Proposition 2.1 yields the conjectured bound on $L^{\tilde{p}}\left(\mathbb{R}^{d}\right)$ when applied to the conjectured bound on $L^{p}\left(\mathbb{R}^{d-1}\right)$.

Proof of (5). Observing that if

$$
p=\frac{d-1}{m} \text { then } \tilde{p}=\frac{(d+1)-1}{m+1},
$$

we start from the bound

$$
\left\|\mathcal{M}_{\delta}^{1} f\right\|_{L^{(n-1)}\left(\frac{4 n}{7}\right)^{\prime}} \lesssim \delta^{-\left(\frac{3}{4}+\epsilon\right)}\|f\|_{L^{\frac{4 n}{7}}\left(\mathbb{R}^{n}\right)}
$$

with $n=d-(k-1)$, which is weaker but more convenient for numerology than (2). Since (11) satisfies the left side of (10) with $m=\frac{7}{4}$ and $d=n+1$, we obtain (5) after $k-1$ iterations of Proposition 2.1.

For a larger improvement in $\alpha$, one may interpolate the known $L^{p}$ bound for $\mathcal{M}_{\delta}^{k-1}$ with the trivial $L^{\infty}$ bound and apply Proposition 2.1 to the resulting $L^{d+1}$ bound. This allows us to use the maximum value, 2, of $\frac{r}{p}$ permitted by Drury and Christ's bound, and yields the following corollary.

Corollary 2.1. Under the assumptions of Proposition 2.1, we may also take $\tilde{p}=\frac{d+1}{2}$, $\tilde{\alpha}=\frac{\alpha}{2}$, and $\tilde{q}=\min \left(\frac{(d+1) q}{p},(d+1)\right)$.

Due to the interpolation, Corollary 2.1 cannot yield a bound for which $\alpha$ is sharp with respect to $p$ as in Conjecture 1.2.

Proof of (6). Starting from (2) with $n=d-(k-1)$, we iteratively apply Corollary $2.1(k-1)$ times to obtain $(6)$.

We would like to point out that the proof of Proposition 2.1 and Corollary 2.1 is similar in spirit to Bourgain's recursive metric entropy estimate in the sense that a more efficient version of his technique, namely the proof of Proposition 3.1 in [12], could be used to derive the localized non-endpoint version of the $L^{\frac{d+1}{2}} \rightarrow L^{d+1} x$-ray transform bound. The idea of expressing an average over a $k$-plane as the average over a $k$-1-plane of the $x$-ray transform and then "unraveling" the integration over $G(d, k)$ into a product integral over $\mathbb{S}^{d-1}$ and $G(d-1, k-1)$ is also due to Bourgain, as he used it in Propositions 3.3 and 3.20 of [2]. There, he gave bounds for $\mathcal{N}^{k}$ with $(d, k)=(4,2)$ and $(d, k)=(7,3)$. We state a generalization of these results below.

\section{The $L^{2}$ method}

Reducing $\alpha$ by a factor of two, as in Corollary 2.1, is not a substantial gain for small $\alpha$. By using an $L^{2}$ estimate of the $x$-ray transform which takes advantage of cancellation, instead of the $L^{\frac{d+1}{2}}$ bound, we may take $\tilde{\alpha}=\alpha-1$ when $\alpha \geq 1$ and obtain a bound for $\mathcal{N}^{k}$ when $\alpha<1$. 
Proposition 3.1. Suppose $k, p \geq 2$ and that a bound for $\mathcal{M}_{\delta}^{k-1}$ on $L^{p}\left(\mathbb{R}^{d-1}\right)$ of the form

$$
\left\|\mathcal{M}_{\delta}^{k-1} f\right\|_{L^{p}(G(d-1, k-1))} \lesssim \delta^{-\frac{\alpha}{p}}\|f\|_{L^{p}\left(\mathbb{R}^{d-1}\right)}
$$

is known. Then if $\alpha \geq 1$ we have the bound

$$
\left\|\mathcal{M}_{\delta}^{k} f\right\|_{L^{p}(G(d, k))} \lesssim \delta^{-\frac{\alpha-1}{p}}\|f\|_{L^{p}\left(\mathbb{R}^{d}\right)}
$$

for $f \in L^{p}\left(\mathbb{R}^{d}\right)$. If $\alpha<1$ we have the bound

$$
\left\|\mathcal{N}^{k} f\right\|_{L^{p}(G(d, k))} \lesssim\|f\|_{L^{p}\left(\mathbb{R}^{d}\right)}
$$

for $f \in L^{p}\left(\mathbb{R}^{d}\right)$ supported on $B(0,1)$.

Before proving the proposition, we give its applications.

Proof of Theorem 1.1. We start from the bound (6) with $d_{0}=d-2-j$ and $k_{0}=$ $k-2-j$. This gives

$$
\alpha_{0}=\frac{3(d-k)}{7 \cdot 2^{k-3-j}}+\epsilon, p_{0}=\frac{d_{0}+1}{2}, \text { and } q_{0}=d_{0}+1 .
$$

The condition $k-j>k_{c r}(d-j)$ ensures that $\alpha_{0}<2$, and so no further improvement in $\alpha$ is necessary. Thus, we use our $j$ "spare" iterations to improve $p$. We note that, in Proposition 2.1, when $m \leq d$,

$$
p \leq \frac{d}{m} \text { implies that } \tilde{p} \leq \frac{d+1}{m+1} .
$$

Since $p_{0}$ satisfies the left inequality in (16) with $m=2$ and $d=d_{0}+1$, we see that we may take

$$
p_{1}=\frac{d_{1}+1}{3}, q_{1}=d_{0}+1, \text { and } \alpha_{1}=\alpha_{0},
$$

where $d_{1}=d_{0}+1=d-2-(j-1)$ and $k_{1}=k_{0}-1=k-2-(j-1)$. Above, we ignore the improvement in $\alpha$ and, through interpolation, we ignore some slight additional improvement in $p$. After $j-1$ further iterations, we have

$$
p_{j}=\frac{d_{j}+1}{2+j}, q_{j}=d_{0}+1, \text { and } \alpha_{j}=\alpha_{0},
$$

where $d_{j}=d-2$ and $k_{j}=k-2$. Applying (13) to (17), and then applying (14) to the result, we obtain (4).

Proof of (7). We obtain (7) by starting from (6) with $d_{0}=d-1$, and $k_{0}=k-1$ (In the case $k=2$, we would simply start from (2)). We then apply (13) once.

The main estimate needed to derive Proposition 3.1 was proven by Smith and Solmon in [14].

Lemma 3.1. For $d \geq 3$

$$
\left\|f_{\xi}(y)\right\|_{L_{\xi, y}^{2}\left(\mathbb{S}^{d-1} \times \mathbb{R}^{d-1}\right)}=C_{d}\|f\|_{\dot{H}^{-\frac{1}{2}}\left(\mathbb{R}^{d}\right)}
$$

where $C_{d}$ is a fixed constant depending only on $d$ and $\dot{H}$ denotes the homogeneous $L^{2}$ Sobolev space. 
It immediately follows that if the Fourier transform $\hat{g}$ of a function $g$ is identically 0 on $B(0, R)$ then

$$
\left\|g_{\xi}(y)\right\|_{L_{\xi, y}^{2}\left(\mathbb{S}^{d-1} \times \mathbb{R}^{d-1}\right)} \lesssim R^{-\frac{1}{2}}\|g\|_{L^{2}\left(\mathbb{R}^{d}\right)} .
$$

To effectively apply (18), we use a Littlewood-Paley decomposition. Let $\phi_{0}$ be a Schwartz function with $\hat{\phi}_{0} \equiv 1$ on $B(0,1)$ and with $\hat{\phi}_{0}$ supported on $B(0,2)$. For $j>0$, define $\phi_{j}=2^{j d} \phi_{0}\left(2^{j} \cdot\right)-2^{(j-1) d} \phi_{0}\left(2^{j-1}\right.$. $)$ so that $\hat{\phi}_{j}$ is supported on $B\left(0,2^{j+1}\right) \backslash$ $B\left(0,2^{j-1}\right)$. Functions are decomposed

$$
f=\sum_{j=0}^{\infty} f_{j}
$$

where $f_{j}=f * \phi_{j}$.

Our last ingredients are two Schwartz-tail estimates needed to reconcile the localization properties of the space and frequency variables.

Lemma 3.2. Suppose $g \geq 0$ and $\hat{\tilde{g}}=\hat{g}$ on $B\left(0, \frac{1}{\delta}\right)$. Then

$$
\mathcal{M}_{\delta}^{k-1}[g] \lesssim \mathcal{M}_{\delta}^{k-1}[|\tilde{g}|]
$$

Proof. For $1 \leq n \leq d$ let $\Phi^{n}$ be a nonnegative Schwartz function on $\mathbb{R}^{n}$ such that $\Phi^{n} \geq 1$ on $B(0,1)$ and $\hat{\Phi}^{n}$ is supported on $B\left(0, \frac{1}{\sqrt{2}}\right)$. For $L \in G(d, k)$ let

$$
\pi_{L, \delta}(x)=\Phi^{k}\left(\operatorname{proj}_{L}(x)\right) \delta^{-(d-k)} \Phi^{d-k}\left(\operatorname{proj}_{L^{\perp}}\left(\frac{x}{\delta}\right)\right) .
$$

Now, define

$$
\widetilde{\mathcal{M}}_{\delta}^{k}[f](L)=\sup _{a \in \mathbb{R}^{d}} \int_{\mathbb{R}^{d}} \pi_{L, \delta}(x-a) f(x) d x .
$$

By construction, $\pi_{L, \delta}(\cdot-a) \gtrsim \frac{\chi_{L_{\delta}(a)}}{\mathcal{L}^{d}\left(L_{\delta}(a)\right)}$ and $\hat{\pi}_{L, \delta}$ is supported on $B\left(0, \frac{1}{\delta}\right)$. Thus

$$
\mathcal{M}_{\delta}^{k}[g] \lesssim \widetilde{\mathcal{M}}_{\delta}^{k}[g]=\widetilde{\mathcal{M}}_{\delta}^{k}[\tilde{g}]
$$

Since $\Phi^{k}$ and $\Phi^{d-k}$ are Schwartz functions, we have

$$
\Phi^{k} \leq \sum_{j=1}^{\infty} c_{j} \chi_{B\left(y_{j}, \frac{1}{2}\right)}
$$

and

$$
\Phi^{d-k} \leq \sum_{j=1}^{\infty} d_{j} \chi_{B\left(z_{j}, \frac{1}{2}\right)}
$$

for some $\left\{c_{j}\right\},\left\{d_{j}\right\} \in l^{1}(\mathbb{N}),\left\{y_{j}\right\} \subset \mathbb{R}^{k}$, and $\left\{z_{j}\right\} \subset \mathbb{R}^{d-k}$. Then, for an appropriately chosen $\left\{a_{j, l}\right\}$

$$
\begin{aligned}
\pi_{L, \delta}(x) & \leq \sum_{j, l=1}^{\infty} c_{j} d_{l} \chi_{B\left(y_{j}, \frac{1}{2}\right)}\left(\operatorname{proj}_{L}(x)\right) \delta^{-(d-k)} \chi_{B\left(z_{l}, \frac{1}{2}\right)}\left(\operatorname{proj}_{L^{\perp}}\left(\frac{x}{\delta}\right)\right) \\
& \lesssim \sum_{j, l=1}^{\infty} c_{j} d_{l} \frac{\chi_{L_{\delta}\left(a_{j, l}\right)}}{\mathcal{L}^{d}\left(L_{\delta}\left(a_{j, l}\right)\right)}
\end{aligned}
$$


Thus,

$$
\widetilde{\mathcal{M}}_{\delta}^{k}[\tilde{g}] \lesssim \sum_{j, l=1}^{\infty} c_{j}, d_{l} \mathcal{M}_{\delta}^{k}[|\tilde{g}|] \lesssim \mathcal{M}_{\delta}^{k}[|\tilde{g}|]
$$

Lemma 3.3. Define

$$
\mathcal{N}_{\text {loc }}^{k-1}[g](L)=\sup _{a \in \mathbb{R}^{d}} \int_{a+\left(L \cap B\left(0, \frac{1}{2}\right)\right)} g(x) d x .
$$

Suppose $\hat{g}$ is supported on $B\left(0, \frac{1}{\delta}\right)$. Then

$$
\mathcal{N}_{\text {loc }}^{k-1}[|g|] \lesssim \mathcal{M}_{\delta}^{k-1}[|g|] .
$$

Proof. Since $\hat{g}$ is supported on $B\left(0, \frac{1}{\delta}\right)$,

$$
g=g * \delta^{-d} \phi_{0}\left(\frac{\cdot}{\delta}\right)
$$

and so

$$
\int_{a+\left(L \cap B\left(0, \frac{1}{2}\right)\right.}|g(x)| d x \leq \int_{\mathbb{R}^{d}}\left|\delta^{-d} \phi_{0}\left(\frac{y}{\delta}\right)\right| \int_{a+y+\left(L \cap B\left(0, \frac{1}{2}\right)\right)}|g(x)| d x d y .
$$

Since $\phi_{0}$ is a Schwartz function,

$$
\left|\phi_{0}\right| \leq \sum_{j=1}^{\infty} c_{j} \chi_{B\left(y_{j}, \frac{1}{2}\right)}
$$

for some $\left\{c_{j}\right\} \in l^{1}(\mathbb{N})$ and $\left\{y_{j}\right\} \subset \mathbb{R}^{d}$. Thus

$$
\begin{aligned}
& \int_{\mathbb{R}^{d}}\left|\delta^{-d} \phi_{0}\left(\frac{y}{\delta}\right)\right| \int_{a+y+\left(L \cap B\left(0, \frac{1}{2}\right)\right)}|g(x)| d x d y \\
& \leq \sum_{j=1}^{\infty} c_{j} \delta^{-d} \int_{B\left(\delta y_{j}, \frac{\delta}{2}\right)} \int_{a+y+\left(L \cap B\left(0, \frac{1}{2}\right)\right)}|g(x)| d x d y \\
& \lesssim \sum_{j=1}^{\infty} c_{j} \mathcal{M}_{\delta}^{k-1}[|g|](L) \\
& \lesssim \mathcal{M}_{\delta}^{k-1}[|g|](L) .
\end{aligned}
$$

Proof of Proposition 3.1. We begin by proving (13). Averaging over each $L_{\delta}(a)$ is local and we are proving an $L^{p} \rightarrow L^{q}\left(L^{r}\right)$ bound where $p \leq q \leq r$, so we may assume that $f$ is supported on the unit ball. Additionally, assume that $f$ is nonnegative.

Following the proof of Proposition 2.1, we observe that for $L=\operatorname{span}\left(\xi, T_{\xi}^{-1}(M)\right)$ we have

$$
\mathcal{M}_{\delta}^{k}[f](L) \lesssim \mathcal{M}_{\delta}^{k-1}\left[f_{\xi} \circ T_{\xi}^{-1}\right](M) .
$$

Since $f$ is supported on the unit ball, we may switch the order of integration between convolution and the $x$-ray transform to obtain

$$
\left\|\left(f_{j}\right)_{\xi}(y)\right\|_{L_{\xi, y}^{\infty}} \lesssim\|f\|_{L^{\infty}\left(\mathbb{R}^{d}\right)}
$$


uniformly in $j$. Hence, interpolation with (18) gives

$$
\left\|\left(f_{j}\right)_{\xi}(y)\right\|_{L_{\xi, y}^{p}} \lesssim\left(2^{-j}\right)^{\frac{1}{p}}\|f\|_{L^{p}\left(\mathbb{R}^{d}\right)}
$$

for any $p \geq 2$.

From Lemma 3.2, we obtain

$$
\mathcal{M}_{\delta}^{k-1}\left[f_{\xi} \circ T_{\xi}^{-1}\right](M) \lesssim \sum_{j=0}^{|\log (\delta)|+1} \mathcal{M}_{\delta}^{k-1}\left[\left|\left(f_{j}\right)_{\xi} \circ T_{\xi}^{-1}\right|\right](M) .
$$

Averaging Lemma 3.3 gives, for each $j$,

$$
\mathcal{M}_{\delta}^{k-1}\left[\left|\left(f_{j}\right)_{\xi} \circ T_{\xi}^{-1}\right|\right](M) \lesssim \mathcal{M}_{2^{-j}}^{k-1}\left[\left|\left(f_{j}\right)_{\xi} \circ T_{\xi}^{-1}\right|\right](M) .
$$

Integrating over $G(d, k)$ and combining the bounds (12) and (21) as in the proof of Proposition 2.1, we obtain

$$
\left\|\mathcal{M}_{\delta}^{k} f\right\|_{L^{p}(G(d, k))} \lesssim \sum_{j=0}^{|\log \delta|+1}\left(2^{j}\right)^{\frac{\alpha-1}{p}}\|f\|_{L^{p}\left(\mathbb{R}^{d}\right)} \lesssim \delta^{-\frac{\alpha-1}{p}}\|f\|_{L^{p}\left(\mathbb{R}^{d}\right)}
$$

from (20), (22), and (23), when $\alpha \geq 1$.

Proceeding to the proof of (14), we have $f$ supported on the unit ball and we assume that $f$ is nonnegative, giving

$$
\mathcal{N}^{k}[f] \lesssim \mathcal{N}_{\text {loc }}^{k}[f]
$$

As before,

$$
\mathcal{N}_{\text {loc }}^{k}[f](L) \lesssim \mathcal{N}_{\text {loc }}^{k-1}\left[f_{\xi} \circ T_{\xi}^{-1}\right](M)
$$

and

$$
\mathcal{N}_{\text {loc }}^{k-1}\left[\left|\left(f_{j}\right)_{\xi} \circ T_{\xi}^{-1}\right|\right](M) \lesssim \mathcal{M}_{2^{-j}}^{k-1}\left[\left|\left(f_{j}\right)_{\xi} \circ T_{\xi}^{-1}\right|\right](M),
$$

giving

$$
\left\|\mathcal{N}^{k} f\right\|_{L^{p}(G(d, k))} \lesssim \sum_{j=0}^{\infty}\left(2^{j}\right)^{\frac{\alpha-1}{p}}\|f\|_{L^{p}\left(\mathbb{R}^{d}\right)} \lesssim\|f\|_{L^{p}\left(\mathbb{R}^{d}\right)}
$$

when $\alpha<1$.

\section{Acknowledgements}

I would like to thank my advisor Andreas Seeger for mathematical guidance and for his suggestion of the topics considered in this article. I would also like to thank Dan Oberlin for carefully reading several of the previous drafts, and the anonymous referee for suggesting a reference.

\section{References}

[1] D. Alvarez, Bounds for some Kakeya-type maximal functions, Berkeley thesis (1997) 24-55.

[2] J. Bourgain, Besicovitch type maximal operators and applications to Fourier analysis, Geom. Funct. Anal. 1 (1991), no. 2, 147-187.

[3] J. Bueti, An incidence bound for $k$-planes in $F^{n}$ and a planar variant of the Kakeya maximal function, preprint.

[4] M. Christ, Estimates for the k-plane transform, Indiana Univ. Math. J. 33 (1984), no. 6, 891910.

[5] S. Drury, $L^{p}$ estimates for the X-ray transform, Illinois J. Math. 27 (1983), no. 1, 125-129. 
[6] K.J. Falconer, Continuity properties of k-plane integrals and Besicovitch sets, Math. Proc. Cambridge Philos. Soc. 87 (1980), no. 2, 221-226.

[7] N. Katz and T. Tao, New bounds for Kakeya problems, J. Anal. Math. 87 (2002) 231-263.

[8] I. Laba and T. Tao, An x-ray estimate in $\mathbb{R}^{n}$, Rev Mat. Iberoamericana 17 (2001), no. 2, 375-407.

[9] P. Mattila, Geometry of sets and measures in Euclidean spaces, Cambridge University Press, Cambridge, (1995)

[10] T. Mitsis, Corrigenda: " $(n, 2)$ sets have full Hausdorff dimension", Rev. Mat. Iberoamericana 21 (2005), no. 2, 689-692.

[11] _ Norm estimates for a Kakeya-type maximal operator, Math. Nachr. 278 (2005), no. 9, 1054-1060.

[12] R. Oberlin, A recursive bound for a Kakeya-type maximal operator, arXiv:math.CA/0511646.

[13] K. Rogers, On a planar variant of the Kakeya problem, Math. Res. Lett. 13 (2006), no. 2-3, 199-213.

[14] K. Smith and D. Solmon, Lower dimensional integrability of $L^{2}$ functions, J. Math. Anal. Appl. 51 (1975), no. 3, 539-549.

[15] T. Wolff, An improved bound for Kakeya type maximal functions, Rev. Mat. Iberoamericana 11 (1995), no. 3, 651-674.

[16] T. Wolff, A mixed norm estimate for the X-ray transform, Rev. Mat. Iberoamericana 14 (1998), no. 3, 561-600.

Mathematics Department, University of Wisconsin-Madison, 480 Lincoln Dr, Madison WI 53706

E-mail address: oberlin@math.wisc.edu 